\title{
A scrutiny of hard pion chiral perturbation theory
}

\section{G. Colangelo, M. Procura*, L. Rothen, R. Stucki}

Albert Einstein Center for Fundamental Physics, Institute for Theoretical Physics, University of Bern, Sidlerstr. 5, CH-3012 Bern, Switzerland

\section{J. Tarrús Castellà}

Departament d'Estructura i Constituents de la Matèria and Institut de Ciències del Cosmos Universitat de Barcelona, Diagonal, 647, E-08028 Barcelona, Catalonia, Spain

E-mail: gilbertoditp.unibe.ch, mprocuraditp.unibe.ch,

stucki@itp.unibe.ch, rothenditp.unibe.ch, tarrus@ecm.ub.es

In the recently proposed framework of hard pion chiral perturbation theory, the leading chiral logarithms are predicted to factorize with respect to the energy dependence in the chiral limit. We have scrutinized this assumption in the case of vector and scalar pion form factors $F_{V, S}(s)$ by means of standard chiral perturbation theory and dispersion relations. We show that this factorization property is valid for the elastic contribution to the dispersion integrals for $F_{V, S}(s)$ but it is violated starting at three loops when the inelastic four-pion contributions arise.

The 7th International Workshop on Chiral Dynamics,

August 6-10, 2012

Jefferson Lab, Newport News, Virginia, USA

\footnotetext{
* Speaker.
} 


\section{Introduction}

In ref. [1] Flynn and Sachrajda studied the $K_{\ell 3}$ decay in the framework of $S U(2)$ chiral perturbation theory $(\chi \mathrm{PT})$ and argued that it is possible to predict the coefficient of the leading chiral log even when the final state pion is hard, namely outside the region of applicability of $\chi$ PT. Bijnens and collaborators $[2,3,4]$ then claimed that one can determine such a leading chiral log from a one-loop calculation in standard $\chi \mathrm{PT}$ even in more general processes where the pion is hard. This approach has been referred to as hard pion chiral perturbation theory $(\mathrm{H} \pi \chi \mathrm{PT})$. The arguments of $\mathrm{H} \pi \chi \mathrm{PT}$ have been applied to the pion form factors $F_{V}$ and $F_{S}$ which are known up to two loops [5]. After expanding the two-loop $\chi \mathrm{PT}$ results in the limit $M_{\pi}^{2} / s \ll 1$, it has indeed been found that [4]

$$
F_{V, S}(s)=\bar{F}_{V, S}(s)\left(1+\alpha_{V, S}(s) L\right)+\mathscr{O}\left(M^{2}\right),
$$

in agreement with $\mathrm{H} \pi \chi \mathrm{PT}$. Here $s$ is the momentum transfer squared and $L$ stands for the leading chiral logarithm, defined as ${ }^{1}$

$$
L \equiv \frac{M^{2}}{(4 \pi F)^{2}} \ln \frac{M^{2}}{s} .
$$

The physical pion mass can be written as an expansion in $M^{2}\left(M_{\pi}^{2}=M^{2}+\mathscr{O}\left(M^{4}\right)\right)$ which is proportional to the average up and down quark masses $\hat{m}$ since $M^{2}=2 B \hat{m}$. Here $F$ is the pion decay constant in the chiral limit $\left(F_{\pi}=F+\mathscr{O}\left(M^{2}\right)\right) . \bar{F}_{V, S}(s)$ denote the form factors for vanishing $u$ and $d$ quark masses and the coefficients $\alpha_{V, S}$ are fixed at one loop. Using the dispersive representation of the form factors, we will explain the result in eq. (1.1) and show that factorization is violated by contributions of multipion intermediate states, which start at three loops. For a more detailed discussion we refer to our paper [6].

We denote $F_{V}(s)$ and $F_{S}(s)$ by the common symbol $F(s)$ (unless it is necessary to distinguish them), with normalization $F(0)=1$. Both these form factors are analytic functions in the cut plane $\left[4 M_{\pi}^{2}, \infty\right)$ and satisfy the following once subtracted dispersion relation

$$
F(s)=1+\frac{s}{\pi} \int_{4 M_{\pi}^{2}}^{\infty} d s^{\prime} \frac{\operatorname{Im} F\left(s^{\prime}\right)}{s^{\prime}\left(s^{\prime}-s\right)} .
$$

Unitarity relates in the elastic region the imaginary part of the form factor to the form factor itself and the $\pi \pi$ partial wave with the same quantum numbers:

$$
\operatorname{Im} F(s)=\sigma(s) F(s) t^{*}(s), \quad \sigma(s)=\sqrt{1-\frac{4 M_{\pi}^{2}}{s}} .
$$

When $s$ gets larger than the inelastic threshold, additional cuts involving more than two intermediate pions start to contribute. Correspondingly, the form factor can be split into an elastic and an inelastic part where the distinction between them is made at the diagrammatic level. We call "elastic' the contribution obtained by solving the dispersion relation with imaginary parts arising only from two-pion intermediate states, both for $F(s)$ and for the $\pi \pi$ scattering amplitude in the unitarity relation, eq. (1.4). In the next section we will show that the elastic term factorizes like in eq. (1.1) to all chiral orders (if we neglect inelastic contributions to the $\pi \pi$ partial waves). In sec. 3 we will describe our calculation of the lowest order inelastic contribution given by the four pion intermediate state. This yields leading chiral $\operatorname{logs}$ that do not respect $\mathrm{H} \pi \chi$ PT-factorization.

\footnotetext{
${ }^{1}$ Writing $\ln M^{2} / s$ as $\ln M^{2} / \mu^{2}+\ln \mu^{2} / s$, one can then equivalently define $L$ as a $\mu^{2}$-dependent quantity as done in refs. $[1,4]$ with the second term to be absorbed in the $\mathscr{O}\left(M^{2}\right)$ contribution in eq. (1.1).
} 


\section{The elastic contribution to $F(s)$}

In our approach we apply chiral counting to the dispersion relation ${ }^{2}$. There are two sources of leading chiral logs in eq. (1.3). The terms proportional to $L$ can indeed arise either after the dispersive integration over $s^{\prime}$ or because they are present in the integrand. Let us first consider the chiral logs generated by the dispersive integration which are produced at the lower integration boundary $s=4 M_{\pi}^{2}$. The power of $s^{\prime}$ in $\operatorname{Im} F$ determines the presence of the logarithm. Expanding both the form factor and the $\pi \pi$ amplitude, one obtains

$$
F\left(s, M_{\pi}^{2}\right)=1+\mathscr{O}(s)+\mathscr{O}\left(M^{2}\right), \quad t\left(s, M_{\pi}^{2}\right)=c_{1} M^{2}+c_{2} s+\mathscr{O}\left(s^{2}\right)+\mathscr{O}\left(M^{4}\right),
$$

where $c_{1}$ and $c_{2}$ are constants. Substituting eqs. (2.1) into eq. (1.3) we obtain

$$
F(s)=1+\frac{s}{\pi} \int_{4 M_{\pi}^{2}}^{\infty} d s^{\prime} \frac{\sigma\left(s^{\prime}\right)}{s^{\prime}\left(s^{\prime}-s\right)}\left(c_{1} M^{2}+c_{2} s^{\prime}+\mathscr{O}\left(p^{4}\right)\right) .
$$

We should then evaluate three types of integrals. The first is the well-known loop function $\bar{J}(s)$, which has the following expansion in $M^{2} / s \ll 1$,

$$
\frac{s}{\pi} \int_{4 M_{\pi}^{2}}^{\infty} d s^{\prime} \frac{\sigma\left(s^{\prime}\right)}{s^{\prime}\left(s^{\prime}-s\right)}=16 \pi \bar{J}(s)=\frac{1}{\pi}\left[1+\ln \frac{M^{2}}{-s}+\frac{2 M^{2}}{s}\left(1-\ln \frac{M^{2}}{-s}\right)+\mathscr{O}\left(\frac{M^{4}}{s^{2}}\right)\right] .
$$

Since the chiral logarithms are produced from the lower integration boundary, for the remaining integrals we can introduce an $M_{\pi}$-independent upper cut-off $\Lambda^{2}$, which allows us to interchange integration and expansion for small $M$. The second type of integral is then given by

$$
\frac{s}{\pi} \int_{4 M_{\pi}^{2}}^{\Lambda^{2}} d s^{\prime} \frac{\sigma\left(s^{\prime}\right)}{\left(s^{\prime}-s\right)}=\bar{d}_{1}\left(s, \Lambda^{2}\right)-\frac{2}{\pi} M^{2} \ln \frac{M^{2}}{s}+\mathscr{O}\left(M^{2}\right)
$$

and the third is the integration over terms of $\mathscr{O}\left(p^{4}\right)$. If these are proportional to $s^{2}$ then they are enough suppressed to not generate a leading chiral log. Otherwise these terms go at least like $M_{\pi}^{2}$, producing contributions that are beyond our accuracy. Collecting the various pieces we obtain

$$
F(s)=\bar{F}(s)+16 \pi F^{2}\left(c_{1}-2 c_{2}\right) L+\mathscr{O}\left(M^{2}\right) .
$$

The constants $c_{1}$ and $c_{2}$ are related to the $\pi \pi$ scattering lengths and effective ranges characterizing the threshold expansion [7]. For the vector form factor the relevant parameters are $c_{1}=-a_{1}^{1}+$ $\mathscr{O}\left(M^{2}\right)$, and $c_{2}=a_{1}^{1} / 4+\mathscr{O}\left(M^{2}\right)$. For the scalar form factor we must use instead $c_{1}=a_{0}^{0} / M^{2}-$ $b_{0}^{0}+\mathscr{O}\left(M^{2}\right)$ and $c_{2}=b_{0}^{0} / 4+\mathscr{O}\left(M^{2}\right)$ leading to

$$
\alpha_{V}=16 \pi F^{2} \lim _{M^{2} \rightarrow 0}\left(-\frac{3 a_{1}^{1}}{2}\right)=-1, \quad \alpha_{S}=16 \pi F^{2} \lim _{M^{2} \rightarrow 0}\left(\frac{a_{0}^{0}}{M^{2}}-\frac{3 b_{0}^{0}}{2}\right)=-\frac{5}{2},
$$

which reproduces the known result [5, 4]. From the discussion above, it is clear that the dispersive integration may only generate a chiral $\log$ at $\mathscr{O}\left(p^{2}\right)$. We shall now consider leading chiral logs at higher chiral orders. We are interested in terms proportional to $s^{n-1} L$ at order $p^{2 n}$, which arise

\footnotetext{
${ }^{2}$ Early calculations of the chiral logs in chiral perturbation theory have been made following a similar approach. For more details we refer to our paper [6] and references therein.
} 
only if the integrand itself contains already a chiral log. We can argue in a recursive way using the unitarity relations for the form factors at different chiral orders:

$$
\begin{aligned}
\operatorname{Im} F^{(2)}(s) & =\sigma(s) t^{(2)}(s) \\
\operatorname{Im} F^{(4)}(s) & =\sigma(s)\left[t^{(4) *}(s)+F^{(2)}(s) t^{(2)}(s)\right]
\end{aligned}
$$

The real parts are obtained by performing the dispersive integrals. The one loop result of the form factor is then $F^{(2)}(s)=\bar{F}^{(2)}(s)+\alpha L+\mathscr{O}\left(M^{2}\right)$, where the coefficient of the log was calculated in eq. (2.6). At the next order (i.e. at two loops) the only source of leading chiral logs is the integrand. The term containing $L$ has as coefficient exactly the absorptive part of the form factor at one chiral order lower times $\alpha$. Therefore, at two loops the form factor can be written as

$$
F(s)=\left(1+\bar{F}^{(2)}(s)\right)(1+\alpha L)+\bar{F}^{(4)}(s)+\mathscr{O}\left(M^{2}\right)+\mathscr{O}\left(p^{6}\right) .
$$

i.e. in the form predicted by $\mathrm{H} \pi \chi \mathrm{PT}$. For the elastic contribution to $F(s)$, the same argument can be repeated in the same way order by order. At every new step the terms which could destroy factorization are the contributions to $\operatorname{Im} F^{(2 n)}$ arising from the $\pi \pi$ scattering amplitude at the same order. In ref. [6], using Roy equations [8], we calculated these terms and found that no leading chiral logs appear from the $\pi \pi$ scattering amplitude. However, at order $p^{6}$, the four-pion intermediate states contribute to $\operatorname{Im} F(s)$ and we will now show that these yield leading chiral logarithms which are responsible for the breakdown of factorization, leading to the three-loop result,

$$
F(s)=\left(1+\bar{F}^{(2)}(s)+\bar{F}^{(4)}(s)\right)(1+\alpha L)+\alpha_{\text {inel }}(s) L+\bar{F}^{(6)}(s)+\mathscr{O}\left(M^{2}\right)+\mathscr{O}\left(p^{8}\right) .
$$

\section{The contribution from inelastic channels}

The lowest-order inelastic contribution to $F(s)$ is given by three-loop diagrams with four intermediate pions, see fig. (1). We evaluated them by means of the following dispersion relation with the lower integration boundary given by the four-pion threshold and $\operatorname{Im} F_{\text {inel }}$ written in terms of the phase space integral which follows from unitarity:

$$
F_{\text {inel }}(s)=\frac{s}{2 \pi} \int_{16 M_{\pi}^{2}}^{\infty} d s^{\prime} \frac{1}{s^{\prime}\left(s^{\prime}-s\right)} \int d \Phi_{4}\left(s ; p_{1}, p_{2}, p_{3}, p_{4}\right) F_{4 \pi} \cdot T_{6 \pi}^{*} .
$$

Here $d \Phi_{4}$ is the phase space for four pions of momenta $p_{1}, \ldots, p_{4} . F_{4 \pi}$ denotes the current- $4 \pi$ vertex and $T_{6 \pi}$ is the six-pion scattering amplitude. Chiral logarithms are either produced by the dispersive integration or by the integral in $d \Phi_{4}$. Since $\operatorname{Im} F_{\text {inel }}$ is of $\mathscr{O}\left(p^{6}\right)$, the integration over $s^{\prime}$ cannot yield terms proportional to $s^{2} L$, as can be seen by applying the same arguments as in sec. 2 .

Therefore we need to concentrate only on the phase space integral. Chiral logarithms are produced by integrations over intermediate momenta with mass-dependent boundaries. We find that, in order to calculate the terms proportional to $L$, we can expand the integrand for small $M$ and keep only the relevant terms. We used the following phase space parametrization since it allows us to perform analytical integrations after this expansion:

$$
d \Phi_{4}=\frac{1}{(4 \pi)^{6}} \frac{\lambda^{1 / 2}\left(s, q^{2}, M_{\pi}^{2}\right)}{2 s} \frac{\lambda^{1 / 2}\left(q^{2}, k^{2}, M_{\pi}^{2}\right)}{2 q^{2}} \frac{\lambda^{1 / 2}\left(k^{2}, M_{\pi}^{2}, M_{\pi}^{2}\right)}{2 k^{2}} d \Omega_{k} d \Omega_{q} d \Omega_{s} \frac{d q^{2}}{2 \pi} \frac{d k^{2}}{2 \pi}
$$




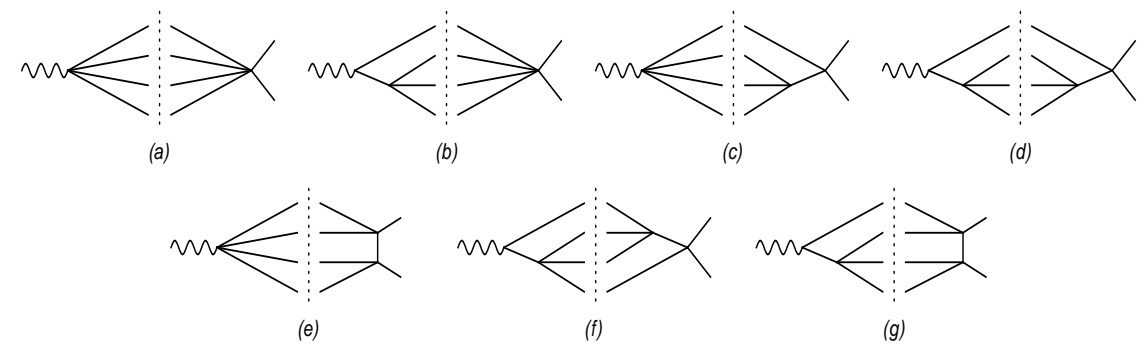

Figure 1: Three-loop cut diagrams contributing to the scalar $F_{\text {inel }}(s)$ at order $p^{6}$.

in terms of the Källén function $\lambda(x, y, z) \equiv x^{2}+y^{2}+z^{2}-2(x y+x z+y z)$. Here $k=p_{1}+p_{2}$ and $q=p_{1}+p_{2}+p_{3} . \Omega_{s}$ is the solid angle in the center-of-mass frame of the two final pions, $\Omega_{q}$ is the solid angle in the frame where $\vec{q}=0$ and $\Omega_{k}$ is the solid angle in the frame where $\vec{k}=0$. The integration range of the kinematic variables $k^{2}$ and $q^{2}$ is determined by the delta function which ensures momentum conservation: $4 M_{\pi}^{2} \leq k^{2} \leq\left(\sqrt{q^{2}}-M_{\pi}\right)^{2}, 9 M_{\pi}^{2} \leq q^{2} \leq\left(\sqrt{s}-M_{\pi}\right)^{2}$. We stress that chiral logarithms stem from both upper and lower integration boundaries due to the functional form of the phase space. More details on this phase space parametrization are given in app. C of our ref. [6]. The advantage of using this parametrization is that each $\lambda$ function contains $M_{\pi}^{2}$ as an argument, which enables us to expand all factors and perform all the integrations analytically, at least for some diagrams.

Let us now consider the scalar form factor. For the diagrams $(a)$ to $(d)$ in fig. (1) we have been able to determine analytically both the values in the chiral limit and the coefficients of $L$ for $\operatorname{Im} F_{\text {inel }}(s)$. The latter ones are given by

$$
\delta_{i} \frac{s^{2}}{(4 \pi F)^{4}} \quad \text { with } \quad \delta_{a}=-\frac{10}{3} \pi, \quad \delta_{b}=\frac{55}{12} \pi, \quad \delta_{c}=\frac{25}{6} \pi \quad \text { and } \quad \delta_{d}=-\frac{9}{4} \pi .
$$

For the remaining diagrams the structure is too complicated to perform all integrations analytically. We computed the corresponding coefficients for the leading chiral logs numerically. With the same numerical routine we were able to reproduce the coefficients $\delta_{i}$ for the diagrams $(a)$ to $(d)$ within one per cent. The contribution from all seven graphs leads to our determination of the coefficient of $L$ in $\operatorname{Im} F_{\text {inel }}(s)$ at three loops:

$$
\delta \frac{s^{2}}{(4 \pi F)^{4}} \quad \text { with } \quad \delta=(-0.53 \pm 0.05) \pi .
$$

The error quoted on $\delta$ is a conservative estimate of the uncertainties in our numerical procedure. After performing the dispersive integral in eq. (3.1), for $\alpha_{\text {inel }}$ in eq. (2.9) we get

$$
\alpha_{\text {inel }}(s)=\left[C\left(\mu^{2}\right)+\frac{\delta}{\pi} \times\left(\ln \frac{\mu^{2}}{s}+i \pi\right)\right] \frac{s^{2}}{(4 \pi F)^{4}}
$$

where $C\left(\mu^{2}\right)$ is a combination of LECs compensating the $\mu$-dependence. Assuming that $C(\mu=$ $1 \mathrm{GeV})$ is of natural size, comparing the logarithms from the elastic and the inelastic part at three loops, we find that the factorization breaking effect is about $5 \%$ in the range for $s$ of interest, i.e. for $\sqrt{s} \ll \Lambda_{\chi}$ where the chiral power counting is applicable. If $\sqrt{s} \simeq \Lambda_{\chi}$, the three-loop contribution is 
not suppressed compared to the two-loop and one-loop results. Furthermore, at $\mathscr{O}\left(p^{8}\right)$ in the form factors there will be contributions from four-pion intermediate cuts in the $\pi \pi$ scattering amplitude $t^{(8)}$. These can be additional sources of chiral logs.

To summarize, our analysis shows that as long as one remains in the chiral regime $M^{2} \ll$ $s \ll \Lambda_{\chi}^{2}$, factorization of the leading chiral log does emerge as a property which holds to a good approximation. However, if we leave the low-energy region, the chiral counting is not valid anymore, higher orders become more and more important and should be taken all into account before drawing any conclusion. Furthermore, if we go to asymptotically large energies, factorization of the chiral log does emerge again as an approximate property, but its origin is quite different and the coefficient of the chiral log gets modified, as we discuss in ref. [6].

\section{Conclusions}

Applying dispersion relations and standard chiral perturbation theory to the calculation of vector and scalar pion form factors, we have shown to what extent the factorization of leading chiral $\operatorname{logs}$ for $M_{\pi}^{2} / s \ll 1$ conjectured in $\mathrm{H} \pi \chi \mathrm{PT}$ is valid. The elastic contribution to the dispersive integrals does lead to factorization while the inelastic part violates it. This implies that starting at three loops $\mathrm{H} \pi \chi \mathrm{PT}$ does not reproduce the correct leading quark mass dependence.

\section{Acknowledgments}

The Albert Einstein Center for Fundamental Physics is supported by the "Innovations- und Kooperationsprojekt C-13" of the "Schweizerische Universitätskonferenz SUK/CRUS". Partial financial support by the Helmholtz Association through the virtual institute "Spin and strong QCD" (VH-VI-231) and by the Swiss National Science Foundation is gratefully acknowledged. JTC acknowledges a MEC FPU fellowship (Spain).

\section{References}

[1] J.M. Flynn and C.T. Sachrajda, SU(2) chiral perturbation theory for K(l3) decay amplitudes, Nucl. Phys. B 812, 64-80 (2009), arXiv:0809.1229.

[2] J. Bijnens and A. Celis, $K \rightarrow \pi \pi$ Decays in SU(2) Chiral Perturbation Theory, Phys.Lett. B 680, 466-470 (2009), arXiv:0906.0302.

[3] J. Bijnens and I. Jemos, Hard Pion Chiral Perturbation Theory for $B \rightarrow \pi$ and $D \rightarrow \pi$ Formfactors, Nucl. Phys. B 840, 54-66 (2010), arXiv:1006.1197.

[4] J. Bijnens and I. Jemos, Vector Formfactors in Hard Pion Chiral Perturbation Theory, Nucl. Phys. B 846, 145-166 (2011), arXiv:1011.6531.

[5] J. Bijnens, G. Colangelo and P. Talavera, The vector and scalar form factors of the pion to two loops, JHEP 05, 14 (1998), hep-ph/9805389.

[6] G. Colangelo, M. Procura, L. Rothen, R. Stucki and J. Tarrús Castellà, On the factorization of chiral logarithms in the pion form factors, JHEP 1209, 131 (2012), arXiv:1208.0498.

[7] J. Gasser and H. Leutwyler, Chiral Perturbation Theory to One Loop, Ann. Phys. 158, 142 (1984).

[8] S.M. Roy, Exact integral equation for pion-pion scattering involving only physical region partial waves, Phys. Lett. B 36, 353 (1971). 